\title{
Differential capacity of human interleukin-4 and interferon- $\alpha$ monocyte-derived dendritic cells for cross-presentation of free versus cell-associated antigen
}

\author{
Jurjen M. Ruben ${ }^{1} \cdot$ Hetty J. Bontkes ${ }^{1} \cdot$ Theresia M. Westers $^{1} \cdot$ Erik Hooijberg $^{2}$ • \\ Gert J. Ossenkoppele ${ }^{1} \cdot$ Tanja D. de Gruijl ${ }^{3} \cdot$ Arjan A. van de Loosdrecht ${ }^{1}$
}

Received: 2 November 2014 / Accepted: 10 July 2015 / Published online: 28 July 2015

(C) The Author(s) 2015. This article is published with open access at Springerlink.com

peptide (SLP) to a MART- $1_{\text {aa26-35 }}$ recognizing $\mathrm{CD}^{+} \mathrm{T}$ cell line, IL-4 MoDC proved more potent cross-primers of antigen-specific $\mathrm{CD} 8^{+} \mathrm{T}$ cells when loaded with blebs. The latter is likely due to the observed greater capacity of IL-4 MoDC to ingest apoptotic blebs. In conclusion, our data indicate the use of IFN $\alpha$ MoDC over IL-4 MoDC in the context of DC vaccination with SLP, whereas IL-4 MoDC are preferred for vaccination with bleb-derived antigens.

Keywords Monocyte-derived dendritic cell · IL-4 · IFN $\alpha \cdot$ Antigen presentation $\cdot$ Vaccination

$\begin{array}{ll}\text { Abbreviations } \\ \text { ACR } & \text { Apoptotic cell remnants } \\ \text { AML } & \text { Acute myeloid leukemia } \\ \text { CFSE } & \text { Carboxyfluorescein succinimidyl ester } \\ \text { CR } & \text { Complement receptor } \\ \text { DC } & \text { Dendritic cells } \\ \text { HLA } & \text { Human leukocyte antigen } \\ \text { IFN } & \text { Interferon } \\ \text { IL } & \text { Interleukin } \\ \text { imMoDC } & \text { Immature MoDC } \\ \text { MART-1 } & \text { Melanoma-associated antigen recognized by } \\ & \text { T cells 1 } \\ \text { matMoDC } & \text { Mature MoDC } \\ \text { MLR } & \text { Mixed leukocyte reaction } \\ \text { MoDC } & \text { Monocyte-derived dendritic cells } \\ \text { PBL } & \text { Peripheral blood lymphocytes } \\ \text { PBMC } & \text { Peripheral blood mononuclear cells } \\ \text { PGE-2 } & \text { Prostaglandin E-2 } \\ \text { SLP } & \text { Synthetic long peptide } \\ \text { TAA } & \text { Tumor-associated antigens } \\ \text { TH } & \text { T helper } \\ \text { TNF } \alpha & \text { Tumor necrosis factors }\end{array}$




\section{Introduction}

Targeting residual leukemic cells using dendritic cell (DC) vaccination is a promising therapeutic strategy for the treatment of acute myeloid leukemia (AML), as well as other types of cancer. Since the use of DC was first proposed for active anti-tumor vaccination by Banchereau and Steinman [1], strategies for loading tumor-associated antigen (TAA) onto DC have been exploited intensively (reviewed for AML in [2, 3]). Loading DC with TAA is an essential step in inducing tumor-directed $\mathrm{T}$ cell responses, and the use of different antigenic sources has proven to affect DC function [2, 4-6], as well as the number of tumor antigen epitopes. Different strategies to load DC with TAA for anti-tumor $\mathrm{T}$ cell priming depend on different (intra)cellular routes of antigen processing. One of the most important capacities of DC in the context of antigen loading is their ability to ingest and cross-present exogenous antigen, a mechanism exploited to load DC with a variety of TAA sources for vaccination purposes. Exogenous antigens [e.g., from apoptotic cells or synthetic long peptides (SLP)] are most efficiently presented in complex with HLA class II [7]. However, DC can present exogenous TAA in HLA class I molecules by means of cross-presentation [8]. The exact mechanism by which cross-presentation occurs and which DC types are most potent in their ability to crosspresent antigen in vivo remains unclear (reviewed in [9]). However, most DC subsets are capable of performing this task and appear to achieve this with similar efficacy [10]. Previously, we have reported that apoptotic blebs, derived from apoptotic AML cells, are a superior source of TAA for the in situ loading of human skin-resident DC, which take up and cross-present TAA from blebs to a higher degree as compared to ACR [11]. Moreover, IL-4 MoDC loaded with isolated apoptotic blebs increased $\mathrm{CD}^{+}{ }^{+} \mathrm{T}$ cell proliferation and $\mathrm{T}$ helper $\left(\mathrm{T}_{\mathrm{H}}\right)$ type I skewing (based on IFN $\gamma$ production), as compared to using ACR; importantly, we have also shown that bleb-loaded IL-4 MoDC induce TAAspecific $\mathrm{CD} 8^{+} \mathrm{T}$ cells with higher avidity [6].

Monocytes are commonly differentiated into MoDC in the presence of GM-CSF and IL-4. Currently, there is growing interest in the use of MoDC differentiated in the presence of GM-CSF and interferon- $\alpha$ (IFN $\alpha$ ), since IFN $\alpha$ MoDC have been described to be more potent in cross-presenting antigen [12-14], which would be beneficial for DCbased tumor vaccines. However, the culture and maturation methods, as well as the antigen sources used in these studies vary. For instance, the culture periods used for the generation of both types of MoDC generally differ, i.e., a 3-day culture period for IFN $\alpha$ MoDC and a 5-day culture period for IL-4 MoDC. In order to synchronize the cultures from the same donors, most studies either increased the IFN $\alpha$
MoDC culture time or shortened IL-4 MoDC differentiation accordingly. Moreover, varying modalities have been used to induce DC maturation in these comparative studies, e.g., TNF $\alpha$, lipopolysaccharides or CD40 ligation [12, 13], as well as different sources of antigen, e.g, soluble protein and apoptotic cells [12-14].

To determine the preferred DC type to serve as a vehicle for AML-derived apoptotic bleb vaccines, we conducted a head-to-head comparison of IL-4 MoDC and IFN $\alpha$ MoDC. These MoDC types were cultured using the commonly used culture protocols (5- vs. 3-day, respectively), matured with a maturation cocktail consisting of IL- $1 \beta$, IL- $6, T N F \alpha$, PGE-2, and subsequently analyzed for their immunophenotype, endocytic capacity for apoptotic blebs and soluble antigen, and their ability to prime naive $\mathrm{T}$ cells, to crosspresent, and to cross-prime antigen-specific $\mathrm{T}$ cells.

\section{Materials and methods}

\section{Generation of apoptotic cell fractions}

In order to facilitate assessment of antigen-specific $\mathrm{CD}^{+}$ $\mathrm{T}$ cell activation and induction, HL60 AML cells were retrovirally transduced with melanoma-associated antigen recognized by $\mathrm{T}$ cells (MART-1), as described previously [15]. Apoptotic cell fractions were generated as described previously [6]. In short, apoptosis was induced by leaving the HL60 cells for $2 \mathrm{~h}$ at $42{ }^{\circ} \mathrm{C}$ in serum-free RPMI (Gibco, Paisley, UK), followed by $\gamma$-irradiation at $5000 \mathrm{rad}$. After 48-72 h, ACR were removed from the preparation by centrifuging at $600 \times g$, after which the resulting supernatant was spun down at $4000 \times g$ to isolate the apoptotic blebs. Next, the blebs were washed with PBS, and the protein concentration was determined using a ND-1000 Nanodrop spectrophotometer (Thermo Fisher Scientific, Breda, the Netherlands). The isolated blebs were stored in liquid nitrogen until use.

\section{Dendritic cell culture}

Monocytes were isolated from peripheral blood mononuclear cells (PBMC) of healthy donors, after informed consent, by magnetically activated cell sorting using CD14 Microbeads (Miltenyi Biotec, Utrecht, the Netherlands). Isolated monocytes were cultured in the presence of $800 \mathrm{U} / \mathrm{ml}$ GM-CSF (Peprotech, the Netherlands), supplemented with either 500 $\mathrm{U} / \mathrm{ml}$ IL-4 (Peprotech, the Netherlands) for the generation of IL-4 MoDC, or $1000 \mathrm{U} / \mathrm{ml}$ IFN $\alpha$ A/D (R\&D Systems) for the induction of IFN $\alpha$ MoDC. IL-4 MoDC were cultured for 5 days and IFN $\alpha$ MoDC for 3 days, as most frequently described in literature [12-14, 16]. 


\section{Dendritic cell immunophenotype, cytokine production, and loading}

After differentiation, MoDC were isolated and the immunophenotype was determined by flow cytometry, using FITC-labeled, PE-labeled, APC, Horizon V450, or PeCy7-labeled antibodies against HLA-ABC, HLADR, CD1a, CD14, CD36, CD40, CD80, CD83, CD86, CLEC9a, Lox-1, CD18/CD11b (complement receptor 3), and CD18/CD11c (complement receptor 4) (all obtained from $\mathrm{BD}$ Biosciences), and the expression levels were subsequently analysed using flow cytometry (LSRFortessa ${ }^{\mathrm{TM}}$, BD Biosciences); the data were analyzed using FACS Diva software (BD Biosciences). MoDC cytokine production was analyzed, after overnight co-culture with irradiated CD40 ligand-expressing J558 cells and $1000 \mathrm{U} / \mathrm{ml} \mathrm{IFN \gamma}$ (Sanquin, Amsterdam, the Netherlands), using an inflammatory cytokine bead array (BD Biosciences, Breda, the Netherlands). For MoDC loading, $2 \times 10^{5} \mathrm{MoDC}$ were loaded with $40 \mu \mathrm{g}$ of blebs in the presence of the differentiation cytokine cocktails (GM-CSF/IL-4, or GM-CSF/IFN $\alpha$ ), and $1 \mathrm{~h}$ after initiating loading, maturation was induced by IL-1 $\beta$ (10 ng/ $\mathrm{ml}), \mathrm{TNF} \alpha(200 \mathrm{U} / \mathrm{ml}$, both from Sanquin, Amsterdam, the Netherlands), IL-6 (10 ng/ml, R\&D systems, Abingdon, UK), and PGE2 (10 ng/ml, Sigma-Aldrich, Zwijndrecht, the Netherlands).

In order to determine uptake, $40 \mu \mathrm{g}$ blebs were labeled with $0.5 \mu \mathrm{M}$ carboxyfluorescein succinimidyl ester (CFSE) (Invitrogen, Breda, the Netherlands), and cultured overnight with $2 \times 0^{5}$ PKH26 red-labeled $(1 \mu \mathrm{M}$, SigmaAldrich) MoDC. The percentage of double-positive cells was analyzed using flow cytometry (LSRFortessa ${ }^{\mathrm{TM}}$ ), as a measure of uptake.

Endocytosis of soluble proteins was analyzed by adding either dextran-FITC ( $2 \mu \mathrm{g} / \mathrm{ml}$, Sigma-Aldrich) or Lucifer Yellow ( $2 \mu \mathrm{g} / \mathrm{ml}$, Sigma-Aldrich) to immature IL-4 or IFN $\alpha$ MoDC for $1 \mathrm{~h}$, after which the uptake was analyzed using flow cytometry (LSRFortessa ${ }^{\mathrm{TM}}$ ).

\section{Mixed leukocyte reaction}

Peripheral blood lymphocytes (PBL) were isolated after informed consent from PBMC of healthy donors, by depleting CD14 ${ }^{+}$cells using CD14 Microbeads (Miltenyi Biotec). PBL were stored in liquid nitrogen until further use. PBL were labeled with $1 \mu \mathrm{M}$ CFSE (Invitrogen) and plated in a 96-well plate at $1 \times 10^{5}$ per well. Mature blebloaded MoDC were added to the wells at DC/PBL ratios of $1: 5,1: 10$, or $1: 20$, and $\mathrm{CD}^{+}{ }^{+} \mathrm{CD} 4^{+}$and $\mathrm{CD} 3^{+} \mathrm{CD} 8^{+} \mathrm{T}$ cell proliferations were analyzed using flow cytometry after 6 days. The day 6 supernatant was analyzed for T cell cytokines, using a TH1/TH2/TH17 cytokine bead array (BD Biosciences, Breda, the Netherlands).

\section{Antigen cross-presentation}

HLA-A2 ${ }^{+}$MoDC were loaded with different concentrations of a 25-mer MART- $1_{\text {aa16-40L }}$ SLP for $2 \mathrm{~h}$, after which MoDC maturation was induced by adding IL- $1 \beta$, IL-6, $\mathrm{TNF} \alpha$, and PGE-2. Blebs were loaded as described above, at $40 \mu \mathrm{g}$ per $2 \times 10^{5} \mathrm{MoDC}$. After loading SLP or blebs overnight, MoDC were harvested and co-cultured for $5 \mathrm{~h}$ with a MART- $1_{\text {aa25-36 }}$ recognizing $\mathrm{CD}^{+} \mathrm{T}$ cell line (MART-1 T cell line, $>95 \%$ pure), in the presence of $1 \mu \mathrm{l} /$ $\mathrm{ml}$ GolgiStop $^{\mathrm{TM}}$ (BD Biosciences). Next, the cells were washed and stained with an APC-labeled MART- $1_{\text {aa26-35 }}$ HLA-A2 dextramer (Immudex, Denmark) for $15 \mathrm{~min}$, followed by 15 min of staining with Horizon V450 or FITClabeled CD3 and CD8 mAbs (both BD Biosciences). After washing, the cells were fixed and permeabilized using $\mathrm{BD}$ Cytofix/Cytoperm ${ }^{\mathrm{TM}}$ solution (BD Biosciences), following manufacturer's protocol. Finally, intracellular IFN $\gamma$ (BD Biosciences) was stained for $30 \mathrm{~min}$ at $4{ }^{\circ} \mathrm{C}$, after which the cells were washed and IFN $\gamma$ production was analyzed using flow cytometry (LSRFortessa ${ }^{\mathrm{TM}}$, BD Biosciences), as a measure of activation.

\section{$\mathrm{CD8}^{+} \mathrm{T}$ cell priming}

$\mathrm{CD}^{+}{ }^{+}$from HLA-A2 ${ }^{+}$PBMC were differentiated into either IL-4 MoDC (5 days) or IFN $\alpha$ MoDC (3 days), and $\mathrm{CD} 8^{+}$and $\mathrm{CD} 14 \mathrm{CD} 8^{-}$cells were stored in liquid nitrogen for further use. IFN $\alpha$ MoDC or IL-4 MoDC were loaded with blebs or MART- $1_{\text {aa26-35L }}$ peptide, and plated at $1 \times 10^{5}$ per well. Autologous $\mathrm{CD} 8^{+} \mathrm{T}$ cells and irradiated $\mathrm{CD} 14^{-} \mathrm{CD} 8^{-}$cells were added to the loaded MoDC at $1 \times 10^{6}$ per well, supplemented with $20 \mathrm{U} /$ $\mathrm{ml} \mathrm{IL-7,} 10 \mathrm{ng} / \mathrm{ml}$ IL-12 (both Sanquin), and $10 \mathrm{ng} / \mathrm{ml}$ IL-6 (R\&D systems) in a final volume of $2 \mathrm{ml}$ in Yssel's medium [17]. After $24 \mathrm{~h}, 10 \mathrm{ng} / \mathrm{ml}$ IL-10 (eBioscience, Vienna, Austria) was added to the co-cultures. The priming cultures were restimulated after 9 or 11 days, and subsequently every 7 days, with freshly loaded (thawed) IFN $\alpha$ MoDC or IL-4 MoDC, respectively. The percentage of dextramer-positive cells was analyzed before each restimulation.

\section{$T$ cell avidity assay}

JY cells were loaded for $3 \mathrm{~h}$ with MART- $1_{\text {aa26-35L }}$ peptide in serum-free RPMI, at concentrations ranging from $10 \mu \mathrm{M}$ to $1 \mathrm{pM}$, in the presence of $3 \mu \mathrm{g} / \mathrm{ml} \beta 2$-microglobulin. $5 \times 10^{5} \mathrm{CD}^{+}{ }^{+} \mathrm{CD}^{+} \mathrm{T}$ cells were cultured with $5 \times 10^{5}$ 
peptide loaded JY, in the presence of $1 \mu \mathrm{l} / \mathrm{ml} \mathrm{GolgiStop}{ }^{\mathrm{TM}}$ (BD Biosciences) for $5 \mathrm{~h}$, after which intracellular IFN $\gamma$ production was assessed as described above.

\section{Statistical analysis}

Statistical analysis was performed with GraphPad Prism version 5 for Windows (GraphPad Software Inc.), using a paired two-tailed Student's $t$ test, Fisher's exact test, or a Wilcoxon matched-pairs signed-rank test as indicated. Differences with $p$ values $\leq 0.05$ were regarded significant.

\section{Results}

\section{Differences in MoDC phenotype post-differentiation and maturation}

We first assessed MoDC phenotype after a 3- or 5-day differentiation period for IFN $\alpha$ or IL-4 MoDC, respectively (Fig. 1a). IFN $\alpha$ MoDC had significantly higher expression levels of HLA class II $(p=0.04)$, and CD14 $(p=0.0005)$, as compared to IL-4 MoDC. Moreover, IFN $\alpha$ MoDC showed a higher expression of CD80 $(p=0.01)$ and CD86 $(p=0.03)$. Next, we assessed the MoDC phenotype upon maturation induction in the presence of apoptotic blebs. In contrast to the immature state, IL-4 MoDC displayed a significantly higher expression of both HLA class I $(p=0.0006)$ and HLA class II $(p=0.01)$ and CD1a ( $p=0.009$ ) following loading with blebs and subsequent maturation (Fig. 1b). Moreover, IL-4 MoDC had a significantly higher expression of CD40 $(p=0.003)$, CD80 $(p=0.002), \operatorname{CD} 83(p=0.001)$, and $\operatorname{CD} 86(p=0.002)$, whereas IFN $\alpha$ MoDC retained a higher CD14 expression after maturation $(p=0.009)$. After inducing maturation, IFN $\alpha$ MoDC down-regulated CD1a, compared to their immature expression levels $(p=0.02)$, at levels lower than those on mature IL-4 MoDC $(p=0.002)$.

\section{IL-4 MoDC are more potent in their ability to ingest blebs and soluble antigen}

As the ingestion of exogenous antigen by MoDC is essential for the (cross-) priming of antigen-specific $\mathrm{T}$ cells, we analyzed the capacity of IFN $\alpha$ and IL-4 MoDC to phagocytose apoptotic blebs. After overnight co-culture, a significantly higher percentage of IL-4 MoDC had ingested blebs as compared to IFN $\alpha \mathrm{MoDC}$, in an immature (85 vs.
A

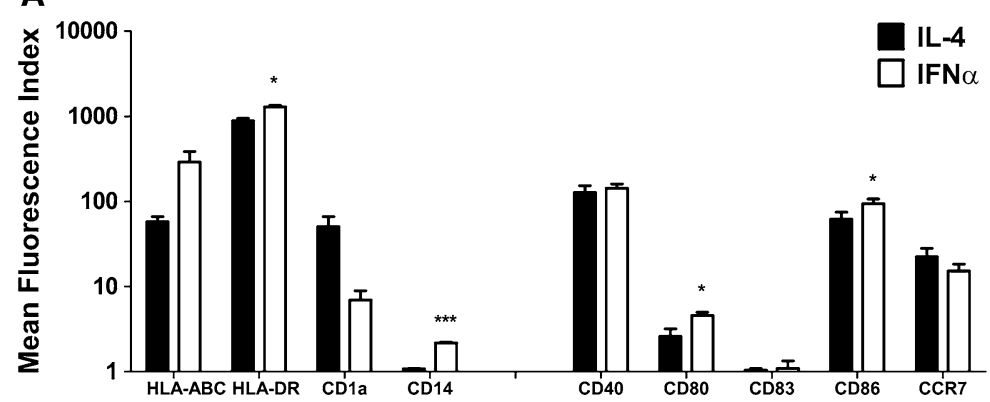

B

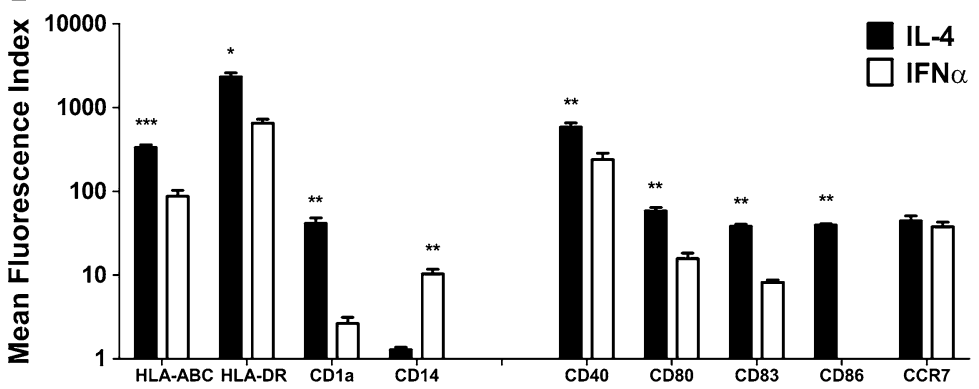

C Apoptotic blebs Pinocytosis Receptor-mediated endocytosis
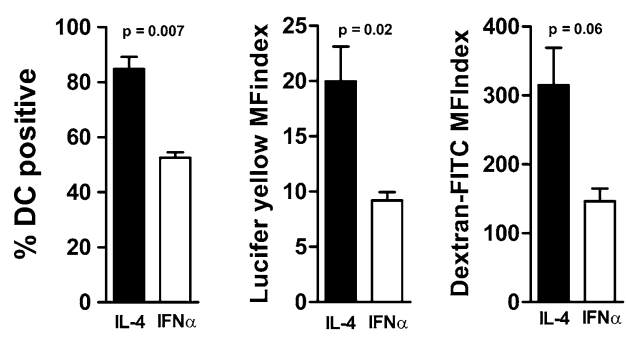

D

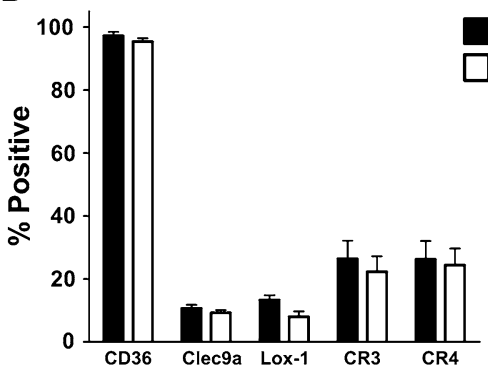

Fig. 1 Analysis of MoDC immunophenotype and endocytic capacity. a Immunophenotype of monocyte-derived dendritic cells (MoDC) generated in the presence of either GM-CSF and IL-4 (black bars), or GM-CSF and IFN $\alpha$ (white bars), for, respectively, 5 or 3 days, $(n=4) . * p<0.05, * * p \leq 0.01, * * * p \leq 0.001$. b Immunophenotype of MoDC $48 \mathrm{~h}$ after loading with blebs and subsequent maturation induction by IL- $1 \beta$, IL- 6 , TNF $\alpha$, and PGE-2 $(n=4) .{ }^{*} p<0.05$, $* * p \leq 0.01, * * * p \leq 0.001$. c Fluorescently labeled MoDC were co- cultured overnight with fluorescently labeled blebs, after which the percentage of double-positive IL-4 (black bars), or IFN $\alpha$ MoDC (white bars), were quantified (apoptotic blebs). Alternatively, MoDC were cultured in the presence of Lucifer Yellow (pinocytosis), or dextran-FITC (receptor-mediated endocytosis). $(n=4)$. d Expression levels of scavenger receptors on immature IL-4 (black bars), or IFN $\alpha$ MoDC (white bars), using flow cytometry $(n=4)$. Statistical significance was for all figures determined using a Student's $t$ test 
$53 \%, p=0.0007$; Fig. 1c, apoptotic blebs) as well as a mature state ( 75 vs., $55 \%, p=0.025$, data not shown). In order to determine whether this difference in the capacity to ingest blebs could be explained by different expression levels of receptors involved in antigen uptake and crosspresentation, we analyzed the expression levels of CD36, Clec9a, Lox-1, complement receptors (CR)3, and CR4 on IFN $\alpha$ versus IL-4 MoDC (Fig. 1d). We observed similar levels of expression of the tested receptors on both immature (Fig. 1d) and mature IFN $\alpha$ and IL-4 MoDC (data not shown). Lectin receptors are described to play a role in apoptotic cell uptake [18], and to assess whether IL-4 MoDC had an increased pinocytosis and lectin receptormediated antigen uptake, we performed a $1 \mathrm{~h}$ co-culture with either Lucifer Yellow (Fig. 1c, pinocytosis) or dextranFITC (Fig. 1c, receptor-mediated endocytosis), respectively. Next, to an increased uptake of blebs, we observed an increased uptake of both Lucifer Yellow $(p=0.02)$ and a near-significant increase in the uptake of dextran-FITC ( $p=0.06$ ) by IL-4 MoDC, as compared to IFN $\alpha$ MoDC.

\section{Cytokine release and allogeneic $\mathbf{T}$ cell induction}

IFN $\alpha$ or IL-4 MoDC were cultured overnight in the presence of IFN $\gamma$ and the CD40 ligand-expressing cell line J558, after which the supernatant was analyzed for inflammatory cytokines. Immature IL-4 MoDC produced significantly higher amounts of IL-6 ( $p=0.04)$ and IL-12p70 ( $p=0.01$ ) (Fig. 2a, immature) as compared to IFN $\alpha$ MoDC. No differences were observed in the secreted levels of IL-1 $\beta$, IL-8, IL-10, and TNF $\alpha$, after CD40 ligation of immature MoDC (Fig. 2a, immature). CD40 ligation following MoDC maturation led to a small but significant increase in the production of IL- 8 by IL- 4 MoDC, compared to IFN $\alpha$ MoDC. Mature IFN $\alpha$ MoDC produced marginally higher levels of IL- $1 \beta$ and considerably higher levels of TNF $\alpha$, compared to IL-4 MoDC (Fig. 2a, mature).

To determine the potency of IFN $\alpha$ or IL- 4 MoDC, loaded with blebs, to prime $\mathrm{T}$ cells, we co-cultured fluorescently labeled allogeneic PBL with bleb-loaded mature MoDC at different ratios. After 6 days of co-culture, we analyzed the induced $\mathrm{T}$ cell proliferation (Fig. 2b, c), and analyzed the produced T cell cytokines. Mature IL-4 MoDC induced a significant increase in $\mathrm{CD}^{+} \mathrm{T}$ cell proliferation over mature IFN $\alpha$ MoDC, whereas no significant differences could be detected between immature IFN $\alpha$ and IL-4 MoDC (Fig. 2b). The increase in $\mathrm{T}$ cell proliferation by mature IL-4 MoDC was attributable to the $\mathrm{CD} 4^{+} \mathrm{T}$ cell compartment, since no clear differences in proliferation could be detected within $\mathrm{CD}^{+} \mathrm{T}$ cell compartment (Fig. 2b). Analysis of the cytokines (IL-2, IL-4, IL-6, IL-10, IL-17a, TNF $\alpha$, and IFN $\gamma$ ) produced by T cells after a 6-day co-culture with matured MoDC showed that despite a difference in proliferation, no significant differences were induced in the released $\mathrm{T}$ cell cytokines between the IFN $\alpha$ and IL-4 MoDC co-cultures (Fig. 2d).

\section{IFN $\alpha$ MoDC have an increased capacity to cross-present a MART-1 synthetic long peptide, compared to IL-4 MoDC}

We next determined the capacity of MoDC to cross-present a 25-mer MART-1 SLP (aa16-40L) and MART-1 from blebs. To this end, immature HLA-A2 ${ }^{+}$MoDC were loaded overnight with either blebs (derived from a stably MART1-expressing AML HL60 cell line) or with the MART-1 SLP, and maturation was induced $1 \mathrm{~h}$ post-loading. Loaded and matured MoDC were subsequently co-cultured for $5 \mathrm{~h}$ with a $\mathrm{CD}^{+}$cytotoxic $\mathrm{T}$ cell line, recognizing the MART$1_{\text {aa26-35 }}$ immunodominant epitope in an HLA-A2 restricted fashion (MART-1 CTL), after which the percentage of IFN $\gamma$ producing MART-1 CTL was determined by intracellular staining using flow cytometry. Unfortunately, this methodology proved to lack the sensitivity to assess cross-presentation efficiency of the MART- $1_{\text {aa26-35 }}$ epitope from blebs. While both IL-4 and IFN $\alpha$ MoDC were capable of crosspresenting the MART- $1_{\mathrm{aa} 26-35}$ epitope from the MART-1 SLP, cross-presentation by IFN $\alpha$ MoDC was more efficient (Fig. 3a). The corresponding mean cross-presentation efficiency, depicted as the IC50 value of the maximal IFN $\gamma$ production by the MART-1 CTL line (Fig. 3b), was $1.17 \mu \mathrm{g} /$ $\mathrm{ml}$ for IL-4 as compared to $0.63 \mu \mathrm{g} / \mathrm{ml}$ for IFN $\alpha$ MoDC $(p=0.07)$. These data demonstrate a clear trend toward a more efficient cross-presenting capacity of IFN $\alpha$ MoDC for soluble protein, in line with previous reports $[13,14]$.

\section{IFN $\alpha$ and IL-4 MoDC have similar capacities to prime antigen-specific $\mathrm{CD8}^{+} \mathrm{T}$ cells in vitro}

We next assessed the ability of IFN $\alpha$ versus IL-4 MoDC to

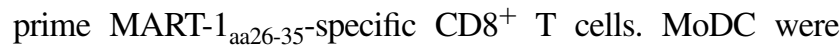
loaded exogenously for $2 \mathrm{~h}$ with the 9-mer MART- $1_{\text {aa26-35L }}$ peptide, after which $\mathrm{CD}^{+} \mathrm{T}$ cells were primed and weekly restimulated with peptide-loaded IFN $\alpha$ and IL-4 MoDC, to analyze antigen-specific priming capacity in an autologous setting. From six healthy donors (six parallel wells per donor), we observed MART-1-specific $\mathrm{CD}^{+} \mathrm{T}$ cell expansion by specific HLA-A2 dextramer staining in 26/36 wells (72\%) and 21/36 wells (58 \%) for peptide-loaded IL-4 MoDC versus IFN $\alpha$ MoDC, respectively (Fig. 4a). In terms of percentage of $\mathrm{CD}^{+} \mathrm{T}$ cells staining dextramer positive, the average percentage per positive well ( $>0.1 \%$ dextramer staining) was higher in the IFN $\alpha$ MoDC versus IL-4 MoDC co-cultures (mean 0.87 vs. $0.43 \%$ respectively, Fig. $4 \mathrm{~b}$ ). Due to high variability, neither of these differences reached statistical significance.

We managed to sort, expand, and test the avidity of IL-4 versus IFN $\alpha$ MoDC-primed MART-1 dextramer-positive 

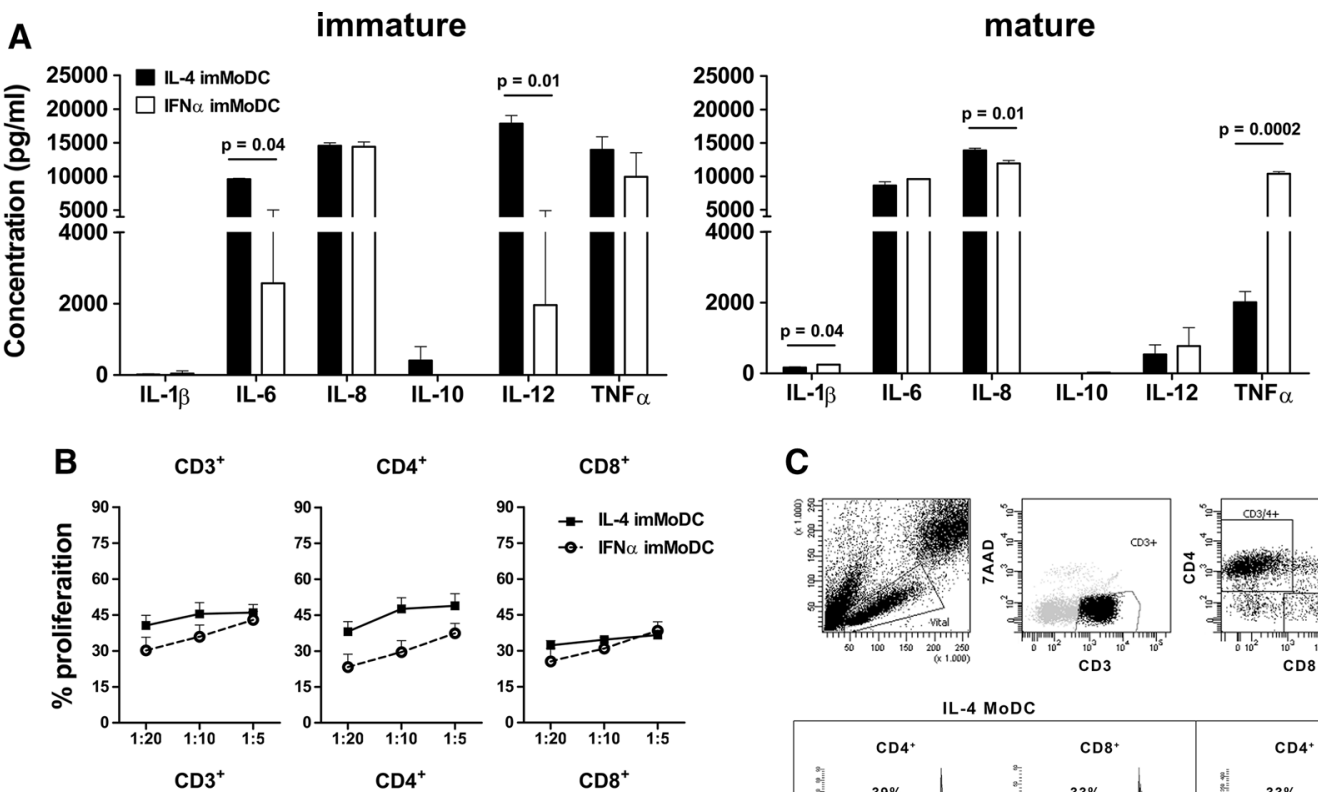

C
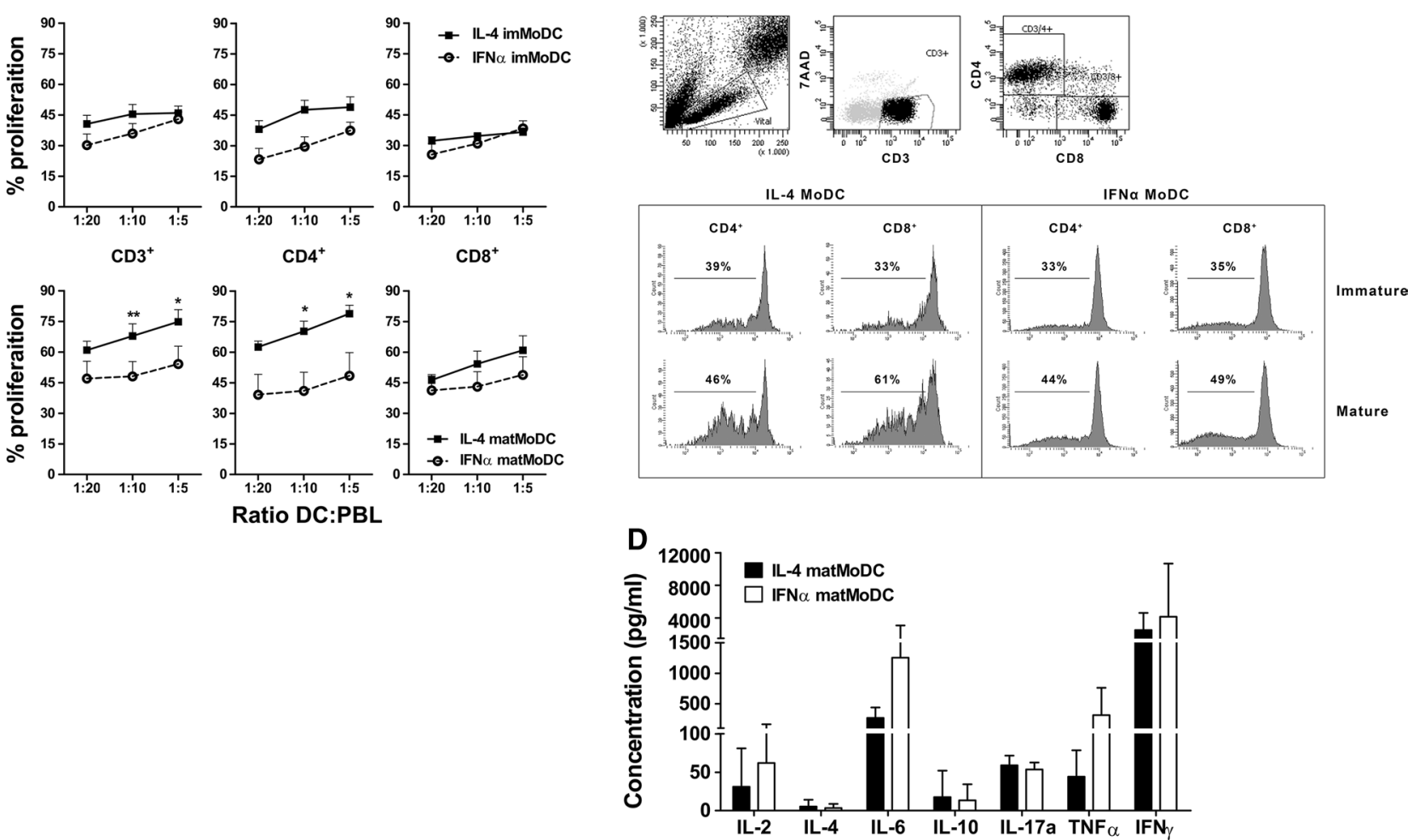

Fig. 2 Cytokine production and $\mathrm{T}$ cell stimulation by MoDC. a Immature MoDC (left graph), or MoDC matured overnight using IL-1 $\beta$, IL-6, TNF $\alpha$, and PGE-2 (i.e., cytokine maturation cocktail, right graph), were ligated with CD40 in the presence of IFN $\gamma$, after which the produced cytokines were analyzed $(n=4)$. b MoDC were loaded with blebs in the absence (imMoDC) or presence (matMoDC) of the cytokine maturation cocktail, and subsequently co-cultured

$\mathrm{CD}^{+} \mathrm{T}$ cells in two out of six donors (Fig. 4c), revealing a higher functional avidity of the IL-4 MoDC-primed T cells in both instances (half max. IFN $\gamma$ production at a mean of 1.9 vs. $57.1 \mathrm{nM}$ peptide for IL-4 and IFN $\alpha$ MoDC-primed $\mathrm{T}$ cells, respectively).

\section{More efficient cross-priming of antigen-specific $\mathrm{CD8}^{+} \mathrm{T}$ cells by bleb-loaded IL-4 MoDC}

We next analyzed the capacity of both MoDC types to cross-prime MART-1 $\mathrm{CD}^{+} \mathrm{T}$ cells, after loading and with fluorescently labeled allogeneic PBL in a mixed leukocyte reaction (MLR). After 6 days, $\mathrm{CD}^{+}$and $\mathrm{CD}^{+} \mathrm{T}$ cell proliferation was analyzed using flow cytometry. $\mathbf{c}$ The gating strategy and representative example of CFSE dilution following a MLR. $\mathbf{d}$ The produced $\mathrm{T}$ cell cytokines were analyzed in the supernatant $(n=4)$. Statistical significance was for all figures determined using a Student's $t$ test

maturing the MoDC overnight with MART-1-expressing HL60 AML blebs. Both MoDC types were capable of subsequent priming of MART- $1_{\text {aa26-35-specific }} \mathrm{CD} 8^{+} \mathrm{T}$ cells. In contrast to peptide-loaded MoDC (Fig. 4a, b), the priming efficiency (mean 94 vs. $53 \%$, Fig. $5 \mathrm{a}, p=0.06$ ) and the mean percentage of MART-1-specific dextramer-positive $\mathrm{CD}^{+} \mathrm{T}$ cells (mean 0.56 vs. $0.12 \%$, Fig. $5 \mathrm{~b}, p \leq 0.05$.) was higher when $\mathrm{CD} 8^{+} \mathrm{T}$ cells were primed with IL-4 MoDC as compared to IFN $\alpha$ MoDC. However, $\mathrm{CD} 8^{+} \mathrm{T}$ cells primed with either MoDC type recognized their cognate antigen with a similar avidity (Fig. 5c); of note, these 


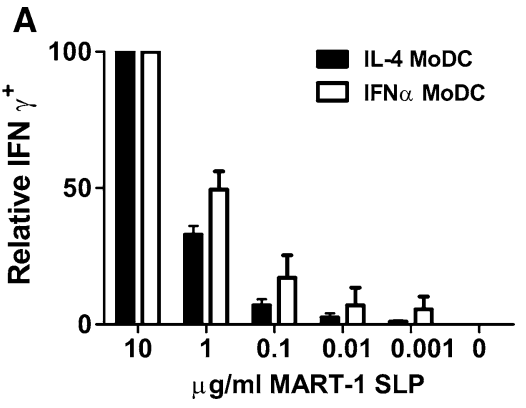

B

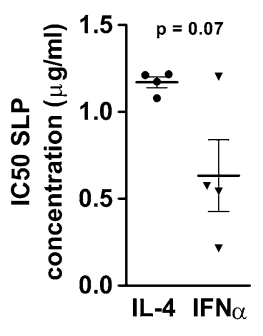

Fig. 3 Cross-presentation of a 25-mer synthetic long peptide (SLP) by MoDC. a Immature IL-4 (black bars) or IFN $\alpha$ MoDC (white bars) were loaded with a titration of MART-1: melanoma-associated antigen recognized by $\mathrm{T}$ cells 1 (MART-1) 25-mer SLP (aa16-40L). After loading and maturing overnight, MoDC were co-cultured with a MART-1 (aa26-35)-specific CD8 ${ }^{+}$T cell line, and intracellular IFN $\gamma$ was quantified. Reactivity data at the highest MART-1 SLP concentration $(10 \mu \mathrm{g} / \mathrm{ml})$ were set to $100 \%$ and were, respectively, 53 and $41 \%$ of the total $\mathrm{CD}^{+}$MART-1 T cells after co-culture with IL-4 or IFN $\alpha \operatorname{MoDC}(n=4)$. b The corresponding cross-presentation efficiency plotted as the SLP concentration, at which $50 \%$ of the $\mathrm{CD}^{+}$ $\mathrm{T}$ cells produced IFN $\gamma$ (IC50), where the percentage of IFN $\gamma$ producing $\mathrm{CD}^{+} \mathrm{T}$ cells at $10 \mu \mathrm{g} / \mathrm{ml}$ was set to $100 \%(n=4)$. Statistical significance was determined using a Student's $t$ test

T cell bulks were derived from the same donors as were the bulk cultures used for avidity analysis upon priming to the 9-mer peptide (Fig. 4c).

\section{Discussion}

The need for targeting multiple TAA or preferably the utilization of an individual's unique mutanome for inducing tumor-directed immunity in vaccination strategies, has become evident in recent years $[19,20]$. Tumor-derived "whole" cellular vaccines have therefore regained interest, especially with the recent insights in immunogenic cell death [21]. We have previously reported on the beneficial effects of using apoptotic AML-derived blebs as a source of TAA for in situ loading of skin DC [11], as well as MoDC loading in vitro [6]. As MoDC cultured in the presence of GM-CSF and IFN $\alpha$ have been described to have a higher capacity for the cross-presentation of antigens [13, 14, 22, 23], they might constitute a more potent platform for the delivery of blebs in the context of DC vaccination strategies. We therefore conducted a head-to-head comparison of these MoDC subtypes.

The expression levels of cell surface antigens found were similar to those described in literature [12, 13], and importantly, loading of blebs did not interfere with maturation of either MoDC type.

Our data clearly show that IL-4 MoDC are superior in taking up exogenous antigen, as the uptake of apoptotic blebs, pinocytosis, and receptor-mediated endocytosis were
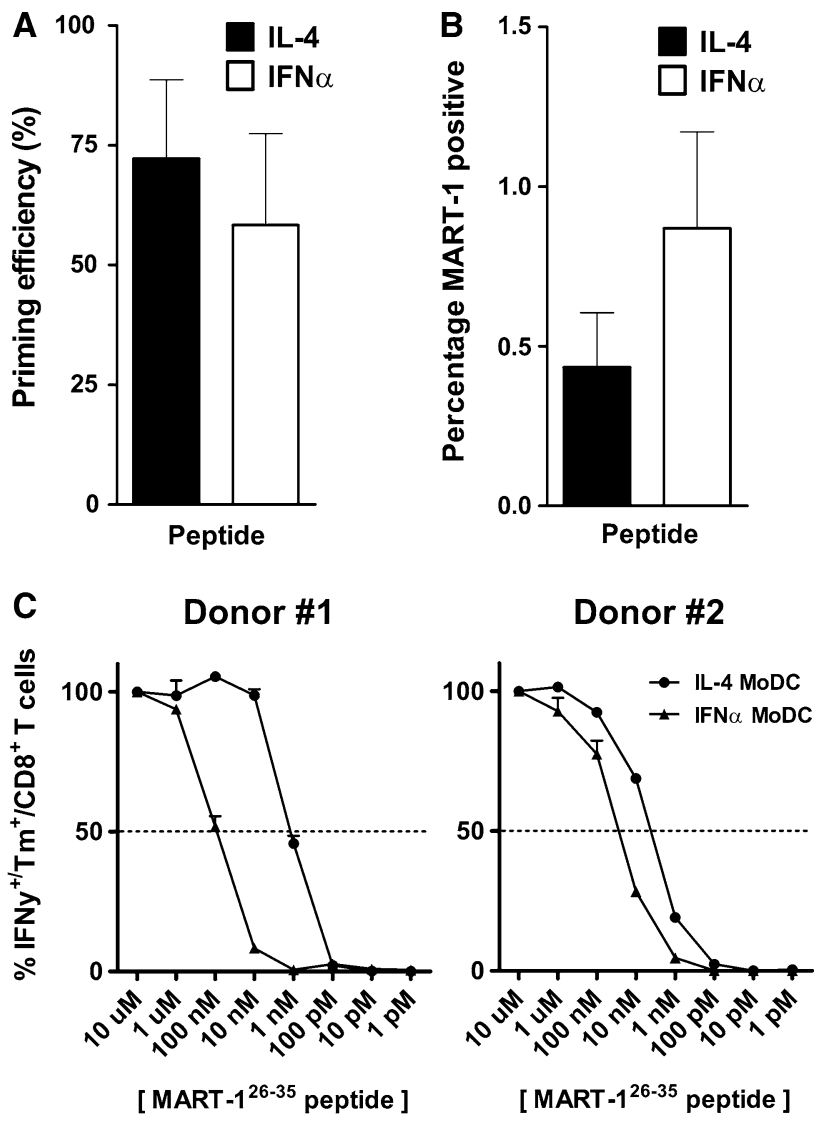

Fig. 4 MoDC priming efficiency and primed $\mathrm{CD}^{+} \mathrm{T}$ cell avidity. a The priming efficiency of six healthy donors was determined by analyzing the number of wells that were scored positive over time, as a percentage of the initiated number of wells, for both IL-4 (black bars) and IFN $\alpha$ MoDC (white bars; $n=36$ ). b The percentages of MART-1 dextramer-positive $\mathrm{CD}^{+}{ }^{+} \mathrm{T}$ cells per well, averaged over six donors. Statistical significance was determined using a Wilcoxon matched-pairs signed-rank test. c MART-1 dextramer-positive cells were sorted and expanded, after which their avidity was analyzed. Shown are the avidity assays of two donors in which we were able to prime, sort, and expand MART- 1 dextramer-positive $\mathrm{CD} 8^{+} \mathrm{T}$ cells to sufficient numbers $(n=2)$

significantly increased, as compared to IFN $\alpha$ MoDC. This difference could not be related to differential expression of receptors previously reported to be involved in the uptake of apoptotic fragments, e.g., LOX-1, which we were unable to detect. The observed lack of LOX-1 expression on IFN $\alpha$ (as well as IL-4) MoDC as compared to earlier studies might be explained by the IFN $\alpha$-subunit and/or source used for differentiation. Whether this also explains the observation that IL-4 MoDC were more potent in ingestion apoptotic blebs remains unclear, since this difference might also be explained by the used antigen source: apoptotic blebs instead of apoptotic cells. Additionally, the modalities used to induce MoDC maturation differ between the conducted studies and ours, which can result in functional differences. It will therefore be of interest to study the 

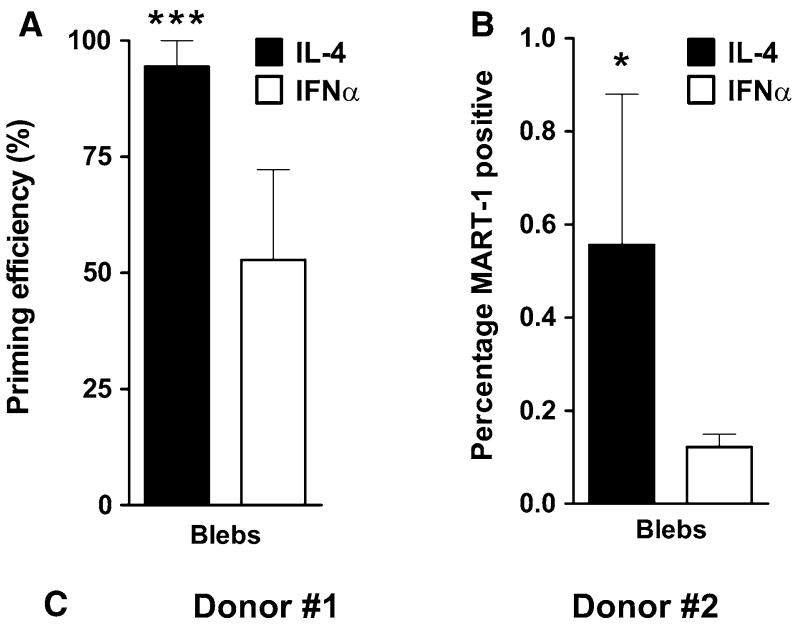

Donor \#2
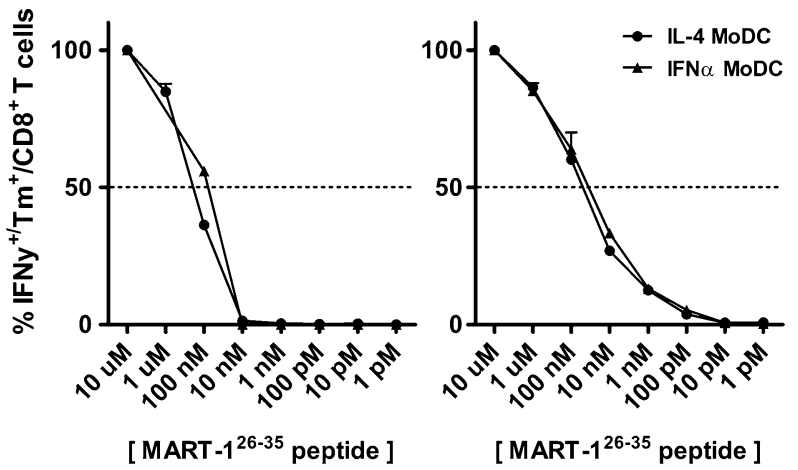

Fig. 5 Cross-priming efficiency of bleb-loaded MoDC and resulting $\mathrm{CD}^{+} \mathrm{T}$ cell avidity. a The priming efficiency in six healthy donors was determined by analyzing the number of wells that were scored positive over time, as a percentage of the initiated number of wells, for both IL-4 (black bars) or IFN $\alpha$ MoDC (white bars; $n=36$, i.e., six parallel wells per condition per donor). ***p $\leq 0.001$ Fisher's exact test. b The percentages of MART-1 dextramer-positive CD8 ${ }^{+}$ $\mathrm{T}$ cells per well, averaged over six donors. ${ }^{*} p \leq 0.05$ Wilcoxon matched-pairs signed-rank test C. MART-1 dextramer-positive cells were sorted and expanded, after which their avidity was analyzed. Shown are the avidity assays of two donors in which we were able to prime, sort, and expand MART- 1 dextramer-positive $\mathrm{CD}^{+}{ }^{+} \mathrm{T}$ cells to sufficient numbers $(n=2)$

functional consequences of different activating stimuli on both types of MoDC in more detail. Moreover, since two types of DC are compared, some of the observed effects do not necessarily reflect a difference in IFN $\alpha$ MoDC, but could also reflect differences in the IL-4 MoDC. These differences in uptake did, however, not account for the observed higher priming efficiency of allogeneic $\mathrm{CD} 4^{+}$ $\mathrm{T}$ cells per se, as unloaded IL-4 MoDC induced a similarly elevated proliferation rate over IFN $\alpha$ MoDC (data not shown). The differences in T cell proliferation by IL-4 MoDC could be a result of the more mature phenotype, in particular the increased expression levels of HLA class II, CD80, and CD83. Since we did not observe striking differences in production of cytokines that we tested (IL-1 $\beta$,
IL-6, IL-8, IL-10, IL-12, and TNF $\alpha$ ) by matured MoDC, this is not likely to result in altered $\mathrm{T}$ cell proliferation, although we did observe more robust production of $\mathrm{TNF} \alpha$ by IFN $\alpha$ MoDC after maturation.

Despite the more efficient uptake of soluble antigens by IL-4 MoDC, IFN $\alpha$ MoDC displayed a higher ability to cross-present the MART- $1_{\text {aa26-35 }}$ epitope derived from a 25-mer MART-1 SLP, similar to what was described previously for OVA [14]. Although we observed a trend toward a higher mean outgrowth percentage of MART1 -specific $\mathrm{CD}^{+} \mathrm{T}$ cells when priming with MART- $1_{\text {aa26- }}$ 35L peptide-loaded IFN $\alpha$ MoDC, the priming efficiency between IL- 4 and IFN $\alpha$ MoDC was similar. However, the avidity of $\mathrm{CD}^{+} \mathrm{T}$ cells that were primed with peptideloaded IL-4 MoDC was higher compared to that of CD8 ${ }^{+}$ $\mathrm{T}$ cells primed with IFN $\alpha$ MoDC (mean of $\sim 2$ vs. $\sim 57 \mathrm{nM}$, respectively). The underlying mechanism leading to the higher avidity remains unclear, but it could be the result of the more mature phenotype of IL-4 MoDC, and possibly traces of the beneficial cytokines (IL-6 and especially IL12 p70 [24]) as observed after CD40 ligation of immature IL-4 MoDC. Although the functional $\mathrm{CD}^{+} \mathrm{T}$ cell avidity between bleb-loaded IL-4 and IFN $\alpha$ MoDC was very similar, a higher percentage of MART-1 positivity was achieved when primed with bleb-loaded IL-4 MoDC.

In this study, we used IFN $\alpha$ as a differentiation-inducing cytokine, but it will be of interest to analyze the effect of adding IFN $\alpha$ in the maturation cytokine cocktail of IL-4 MoDC, on their cross-priming ability, since it has been shown that this has beneficial effects on CCR7 expression and $\mathrm{T}$ cell responses [25].

We show that both IFN $\alpha$ and IL-4 MoDC are efficient primers of $\mathrm{CD}^{+} \mathrm{T}$ cell responses. However, the choice of either MoDC type as vaccination vehicle depends on the antigen source used. SLP-based therapies could benefit from using IFN $\alpha$ MoDC, whereas IL-4 MoDC appear better suited for cross-priming of $\mathrm{CD}^{+} \mathrm{T}$ cells to epitopes derived from apoptotic blebs, most likely due to their superior bleb uptake.

Acknowledgments This work was supported by the Cancer Center Amsterdam/VUmc Institute for Cancer. Immunology Grant: CCA 2007-5-09.

\section{Compliance with ethical standards}

Conflict of interest The authors have no conflicts of interest to report.

Open Access This article is distributed under the terms of the Creative Commons Attribution 4.0 International License (http://creativecommons.org/licenses/by/4.0/), which permits unrestricted use, distribution, and reproduction in any medium, provided you give appropriate credit to the original author(s) and the source, provide a link to the Creative Commons license, and indicate if changes were made. 


\section{References}

1. Banchereau J, Steinman RM (1998) Dendritic cells and the control of immunity. Nature 392:245-252. doi:10.1038/32588

2. Ruben JM, Visser LL, Bontkes HJ et al (2013) Targeting the acute myeloid leukemic stem cell compartment by enhancing tumor cell-based vaccines. Immunotherapy 5:859-868. doi:10.2217/imt.13.76

3. Van den Ancker W, Van Luijn MM, Westers TM et al (2010) Recent advances in antigen-loaded dendritic cell-based strategies for treatment of minimal residual disease in acute myeloid leukemia. Immunotherapy 2:69-83. doi:10.2217/imt.09.85

4. Henry F, Boisteau O, Bretaudeau L et al (1999) Antigen-presenting cells that phagocytose apoptotic tumor-derived cells are potent tumor vaccines. Cancer Res 59:3329-3332

5. Liu K, Iyoda T, Saternus $M$ et al (2002) Immune tolerance after delivery of dying cells to dendritic cells in situ. J Exp Med 196:1091-1097. doi:10.1084/jem.20021215

6. Ruben JM, van den Ancker W, Bontkes HJ et al (2014) Apoptotic blebs from leukemic cells as a preferred source of tumor-associated antigen for dendritic cell-based vaccines. Cancer Immunol Immunother 63(4):335-345. doi:10.1007/ s00262-013-1515-6

7. Sant AJ (1994) Endogenous antigen presentation by MHC class II molecules. Immunol Res 13:253-267

8. Brossart P, Bevan MJ (1997) Presentation of exogenous protein antigens on major histocompatibility complex class I molecules by dendritic cells: pathway of presentation and regulation by cytokines. Blood 90:1594-1599

9. Joffre OP, Segura E, Savina A, Amigorena S (2012) Crosspresentation by dendritic cells. Nat Rev Immunol 12:557-569. doi:10.1038/nri3254

10. Segura E, Durand M, Amigorena S (2013) Similar antigen crosspresentation capacity and phagocytic functions in all freshly isolated human lymphoid organ-resident dendritic cells. J Exp Med 210:1035-1047. doi:10.1084/jem.20121103

11. Ruben JM, Bontkes HJ, Westers TM et al (2014) In situ loading of skin dendritic cells with apoptotic bleb-derived antigens for the induction of tumor-directed immunity. Oncoimmunology 3:e946360. doi:10.4161/21624011.2014.946360

12. Lapenta C, Santini SM, Spada M et al (2006) IFN-alpha-conditioned dendritic cells are highly efficient in inducing crosspriming CD8(+) T cells against exogenous viral antigens. Eur J Immunol 36:2046-2060. doi:10.1002/eji.200535579

13. Parlato S, Romagnoli G, Spadaro F et al (2010) LOX-1 as a natural IFN-alpha-mediated signal for apoptotic cell uptake and antigen presentation in dendritic cells. Blood 115:1554-1563. doi:10.1182/blood-2009-07-234468

14. Spadaro F, Lapenta C, Donati S et al (2012) IFN- $\alpha$ enhances cross-presentation in human dendritic cells by modulating antigen survival, endocytic routing, and processing. Blood 119:1407-1417. doi:10.1182/blood-2011-06-363564

15. Hooijberg E, Ruizendaal JJ, Snijders PJ et al (2000) Immortalization of human $\mathrm{CD} 8^{+} \mathrm{T}$ cell clones by ectopic expression of telomerase reverse transcriptase. J Immunol 165:4239-4245

16. Dauer M, Pohl K, Obermaier B et al (2003) Interferon-alpha disables dendritic cell precursors: dendritic cells derived from interferon-alpha-treated monocytes are defective in maturation and T-cell stimulation. Immunology 110:38-47

17. Yssel H, De Vries JE, Koken M et al (1984) Serum-free medium for generation and propagation of functional human cytotoxic and helper T cell clones. J Immunol Methods 72:219-227

18. Fadok VA, Bratton DL, Henson PM (2001) Phagocyte receptors for apoptotic cells: recognition, uptake, and consequences. J Clin Invest 108:957-962. doi:10.1172/JCI14122

19. Wick DA, Webb JR, Nielsen JS et al (2014) Surveillance of the tumor mutanome by $\mathrm{T}$ cells during progression from primary to recurrent ovarian cancer. Clin Cancer Res 20:1125-1134. doi:10.1158/1078-0432.CCR-13-2147

20. Kreiter S, Castle JC, Türeci O, Sahin U (2012) Targeting the tumor mutanome for personalized vaccination therapy. Oncoimmunology 1:768-769. doi:10.4161/onci.19727

21. Obeid M, Tesniere A, Ghiringhelli F et al (2007) Calreticulin exposure dictates the immunogenicity of cancer cell death. Nat Med 13:54-61. doi:10.1038/nm1523

22. Santini SM, Lapenta C, Donati $S$ et al (2011) Interferon- $\alpha$ conditioned human monocytes combine a Th1-orienting attitude with the induction of autologous Th17 responses: role of IL-23 and IL-12. PLoS One 6:e17364. doi:10.1371/journal.pone.0017364

23. Lattanzi L, Rozera C, Marescotti D et al (2011) IFN- $\alpha$ boosts epitope cross-presentation by dendritic cells via modulation of proteasome activity. Immunobiology 216:537-547. doi:10.1016/j.imbio.2010.10.003

24. Bontkes HJ, Kramer D, Ruizendaal JJ et al (2007) Dendritic cells transfected with interleukin-12 and tumor-associated antigen messenger RNA induce high avidity cytotoxic T cells. Gene Ther 14:366-375

25. Dauer M, Schad K, Junkmann J et al (2006) IFN-alpha promotes definitive maturation of dendritic cells generated by short-term culture of monocytes with GM-CSF and IL-4. J Leukoc Biol 80:278-286. doi:10.1189/jlb.1005592 